\title{
Infection Kinetics and Tropism of Borrelia burgdorferi sensu lato in Mouse After Natural (via Ticks) or Artificial (Needle) Infection Depends on the Bacterial Strain
}

\section{OPEN ACCESS}

Edited by:

Lisa Sedger,

University of Technology Sydney,

Australia

Reviewed by:

Charles Robert Brown, University of Missouri, United States Monica E. Embers,

Tulane University, United States

${ }^{*}$ Correspondence:

Valérie Choumet

vchoumet@pasteur.fr

tThese authors have contributed equally to this work.

¥Present Address:

Elisabeth Ferquel, Unité Environnement et Risques Infectieux, Institut Pasteur, Paris,

France

Specialty section:

This article was submitted to Infectious Diseases,

a section of the journal Frontiers in Microbiology

Received: 19 April 2018 Accepted: 10 July 2018 Published: 31 July 2018

Citation:

Sertour N, Cotté V, Garnier M.

Malandrin L, Ferquel E and Choumet V (2018) Infection Kinetics and Tropism of Borrelia burgdorferi sensu lato in Mouse After Natural (via

Ticks) or Artificial (Needle) Infection Depends on the Bacterial Strain. Front. Microbiol. 9:1722. doi: 10.3389/fmicb.2018.01722

\author{
Natacha Sertour ${ }^{1}$, Violaine Cotté ${ }^{1}$, Martine Garnier ${ }^{1}$, Laurence Malandrin ${ }^{2}$, \\ Elisabeth Ferquel ${ }^{1+\neq}$ and Valérie Choumet ${ }^{1,3 *+}$ \\ ${ }^{1}$ CNR des Borrelia, Institut Pasteur, Paris, France, ${ }^{2}$ BIOEPAR, INRA, Oniris, Université Bretagne Loire, Nantes, France, \\ ${ }^{3}$ Unité Environnement et Risques Infectieux, Institut Pasteur, Paris, France
}

Borrelia burgdorferi sl is a complex of pathogen bacteria transmitted to the host by Ixodes ticks. European Ixodes ricinus ticks transmit different $B$. burgdorferi species, pathogenic to human. Bacteria are principally present in unfed tick midgut, then migrate to salivary glands during blood meal and infect a new host via saliva. In this study, efficiency of transmission in a mouse model of three pathogen species belonging to the $B$. burgdorferi sl complex, B. burgdorferi sensu stricto (B31, N40, and BRE-13), B. afzelii (IBS-5), and $B$. bavariensis (PBi) is examined in order to evaluate infection risk after tick bite. We compared the dissemination of the Borrelia species in mice after tick bite and needle injection. Location in the ticks and transmission to mice were also determined for the three species by following infection kinetics. After inoculation, we found a significant prevalence in the brain for $\mathrm{PBi}$ and $\mathrm{BRE}-13$, in the heart, for $\mathrm{PBi}$, in the skin where B31 was more prevalent than $\mathrm{PBi}$ and in the ankle where both B31 and N40 were more present than PBi. After tick bite, statistical analyses showed that BRE-13 was more prevalent than $\mathrm{N} 40$ in the brain, in the bladder and in the inguinal lymph node. When Borrelia dissemination was compared after inoculation and tick bite, we observed heart infection only after tick inoculation of BRE-13, and PBi was only detected after tick bite in the skin. For N40, a higher number of positive organs was found after inoculation compared to tick bite. All European B. burgdorferi sl strains studied were detected in female salivary glands before blood meal and infected mice within $24 \mathrm{~h}$ of tick bite. Moreover, Borrelia-infected nymphs were able to infect mice as early as $12 \mathrm{~h}$ of tick attachment. Our study shows the need to remove ticks as early as possible after attachment. Moreover, Borrelia tropism varied according to the strain as well as between ticks bite and needle inoculation, confirming the association between some strains and clinical manifestation of Lyme borreliosis, as well as the role played by tick saliva in the efficiency of Borrelia infection and dissemination in vertebrates.

Keywords: lyme borreliosis, Borrelia, Ixodes ricinus, transmission, tick bite, mouse 


\section{INTRODUCTION}

Lyme borreliosis (LB) is the most commonly occurring vectorborne disease in Europe. It is caused by spirochetes belonging to the Borrelia burgdorferi sensu lato (sl) complex. This complex comprises species: Borrelia burgdorferi sensu stricto (ss), B. afzelii, B. garinii, B. bavariensis, B. spielmanii, B. valaisiana and B. lusitaniae (Rijpkema et al., 1997; Collares-Pereira et al., 2004; Rudenko et al., 2011; Stanek et al., 2011). The bacteria are transmitted by a hard tick bite of Ixodes genus. Ticks can parasitize a wide range of hosts. Some of them may be reservoirs for Borrelia, humans being considered as accidental hosts.

In Europe, nearly 85,000 cases are reported each year, an underestimated figure due to the many undeclared or undiagnosed cases. The highest number of cases is reported in Germany, Austria, the Czech Republic, Slovenia and the Balkans (Lindgren and Jaenson, 2006). In France, the LB incidence rate is around 42 per 100,000 inhabitants (Vandenesch et al., 2014). LB is contracted by both forest professionals and recreational visitors.

Lyme borreliosis (LB) can affect a wide range of tissues including skin, the nervous system, joints, heart, and less frequently other organs (Stanek and Reiter, 2011). The most common presenting symptom is erythema migrans, a typical skin lesion that generally appears within 3-30 days subsequently to the infectious tick bite. Days to weeks after infection, B. burgdorferi disseminates through the bloodstream and/or lymphatic system to invade and colonize various tissues, such as the synovial fluid of joints, the heart and the nervous system. Neuroborreliosis is the most common manifestation in Europe and is due to the geographic spread of B. garinii (Stanek and Reiter, 2011; Koedel et al., 2015). Arthritis, a late manifestation targeting large joints, is commonly reported in North America, where B. burgdorferi ss species are dominant. Cardiac manifestations have been reported during both the acute phase and the chronic stage, but is rare $(<5 \%)$. Acrodermatitis chronica atrophicans is associated with Borrelia afzelii (Coipan et al., 2016).

The epidemiological chain of LB comprises three links: natural reservoirs (small mammals, birds), the vector and animal species known to amplify the tick cycle (large mammals). In Europe, the vector of B. burgdorferi $\mathrm{sl}$ is the hard tick Ixodes ricinus. This tick is present especially in humid and forest areas. It is a mandatory bloodsucking parasite, with a development cycle implying three stages (larva, nymph, adult) interspersed with bloodmeals and molts.

I. ricinus is able to feed on more than 240 different species. Reservoirs provide both host and prolonged survival of the pathogen, which increases the likelihood that a tick contracts the bacteria from gorging on the host (Skotarczak, 2009). For most tick-borne pathogens, transmission to the vertebrate host occurs during a blood meal via the saliva of the vector. Both salivary glands and saliva play an important role in the transmission process. Tick saliva contains a large variety of components able to counteract host haemostasis and play a role on the tuning of host immune responses (Šimo et al., 2017). Enhancement of pathogen transmission by tick saliva has been reported for several tick-pathogen associations (Labuda and Nuttall, 2004). Interestingly, the mode of inoculation of B. burgdorferi sl was shown to influence infection in inbred strains of mouse (Gern et al., 1993).

Humans are considered accidental hosts and transmission can occur when in contact with a vector-adapted environment, where vertebrate hosts and their associated ticks are present, or when animals transport ticks into areas of human dwelling. It is usually specified in health agency guidelines that the risk of LB rises with duration of attachment of the tick. In the same way, it is regularly specified that the danger of transmission is very low or absent if the tick is removed within $24-48 \mathrm{~h}$ (Eisen, 2018).

In this study, we were interested in defining kinetics of mouse infection by ticks (nymphs and adults) infected by different Borrelia strains or species. We also compared the dissemination of various Borrelia strains and species in mice infected by various modes of inoculation (via infected ticks or by needle injection).

\section{MATERIALS AND METHODS}

\section{Mice}

Specific-pathogen-free mice C3H/HeN (5-7-week-old females) were purchased from Janvier (St Berthevin, France). Mice were housed in the Institut Pasteur's Animal facilities, accredited by the French Ministry of Agriculture to perform experiments on live mice, in appliance with the French and European regulations on care and protection of Laboratory animals. The protocol of our experiments was approved by the Animal Ethics Committee of Pasteur Institute (CETEA-Institut Pasteur) and by the French Ministry of Higher Education and Research (MESR 00762.02).

\section{Bacterial Strains}

Table 1 listed the B. burgdorferi sensu lato (sl) strains used in this study. They were all cultivated in BSK medium (Sigma) at $33^{\circ} \mathrm{C}$. The number and motility of spirochetes were determined by dark-field microscopy with a Petroff Hausser counting chamber (Hausser scientific). The presence of ospC was verified by PCR using the following primers: forward BRE-13, B31 and N40: AAAAAAAAGGATCCGGAAAAGATG GGAATGC; forward PBi: AAAAAAAAGGATCCGGTGGGG ATTCTGCATC; forward IBS-5: AAAAAAAAGGATCCGGGA AAGGTGGGTC; reverse for all strains and species: AAAAAA AACTCGAGCTAAGGTTTTTTTGGACTTCT TGC.

\section{Infection of Ticks}

I. ricinus nymphs and larvae were obtained from the pathogenfree breeding colony at the UMR BioEpAR (Oniris, France). Ticks were reared and maintained in chambers with a relative humidity of $80-90 \%$ at room temperature before feeding.

Each cohort (200 ticks) was controlled for the absence of B. burgdorferi sl DNA by PCR.

To obtain infected nymphs or adults, larvae and nymphs were allowed to feed on infected mice until repletion 14-28 days after mouse infection, depending on the result of ear biopsies (Figure 1). For each strain, 2 series of 40 larvae or 20 nymphs were allowed to feed per mouse at 1 week interval. Replete ticks were maintained at $20^{\circ} \mathrm{C}$ and $95 \%$ relative humidity and used about 2 months after they molted into potentially infected nymphs and adults. Tick infection rates were evaluated by DNA 
TABLE 1 | Origins of the different B. burgdorferi sl strains used in this study.

\begin{tabular}{|c|c|c|c|c|c|c|c|}
\hline Name & Species & Origin & Source & OspC groups & Preferential tropism & Localization & References \\
\hline B31 & B. burgdorferi ss & North America & I. scapularis & A (Invasive) & Joint & Europe and North America & Fraser et al., 1997 \\
\hline BRE-13 & B. burgdorferi ss & France & $\begin{array}{l}\text { Human } \\
\text { (CSF) }\end{array}$ & Q (invasive) & Joint & Europe & Lagal et al., 2003 \\
\hline $\mathrm{PBi}$ & B. bavariensis & Germany & $\begin{array}{l}\text { Human } \\
\text { (CSF) }\end{array}$ & G4 (Invasive) & Nervous system & Europe & Margos et al., 2009 \\
\hline IBS-5 & B. afzelii & France & $\begin{array}{l}\text { Human } \\
\text { (EM) }\end{array}$ & Invasive & Skin & Europe & - \\
\hline N40 & B. burgdorferi ss & North America & I. scapularis & E (invasive) & Skin & North America & Pachner and Itano, 1990 \\
\hline
\end{tabular}

EM, erythema migrans; CSF, cerebrospinal fluid.

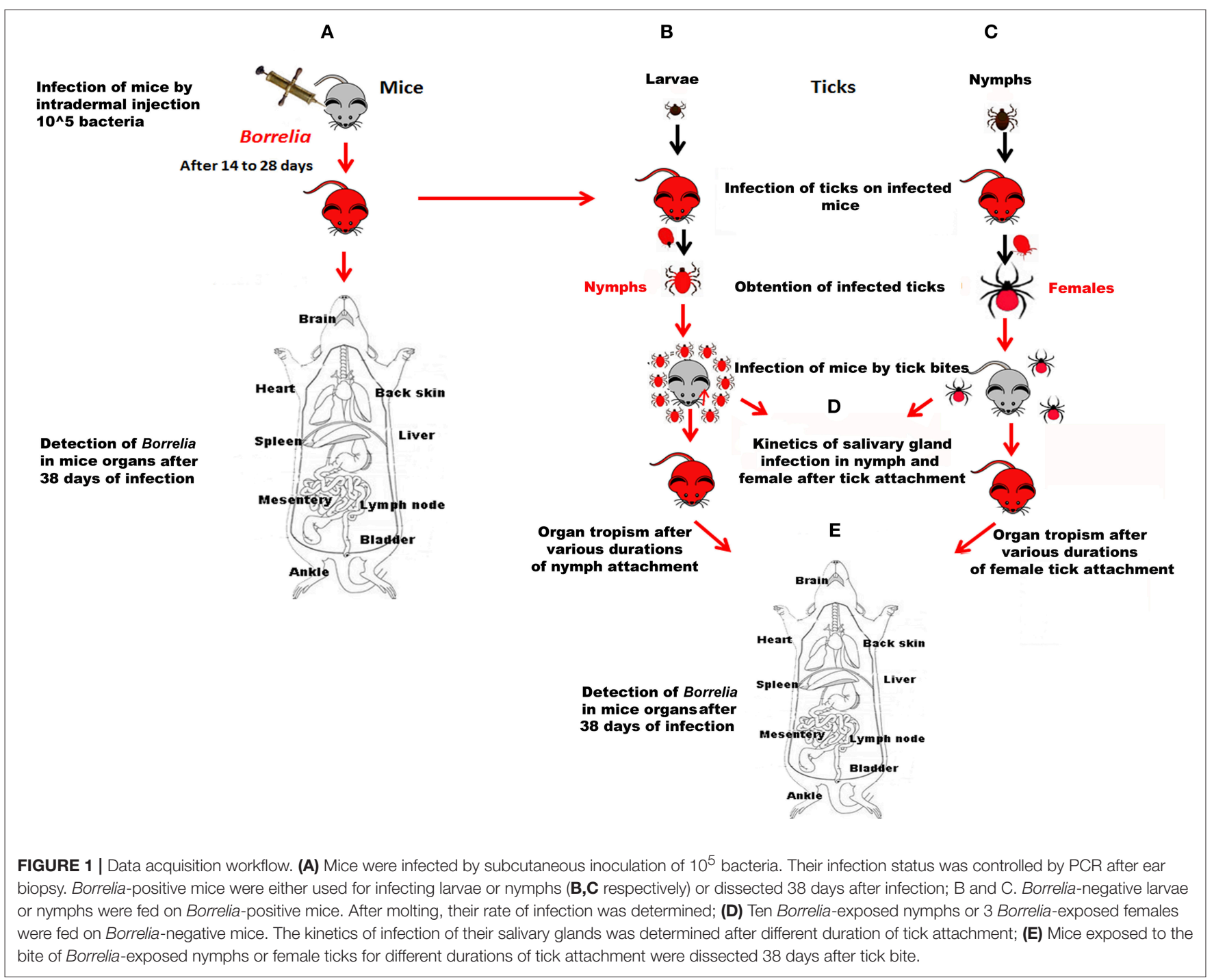

detection on the carcasses of 30 nymphs and on a range of 23-36 adults depending on the Borrelia strain.

\section{Infection of Mice With $B$. burgdorferi sI}

1. Infection of mice by subcutaneous inoculation (Figure 1A). For each strain, ten mice were subcutaneously inoculated with $0.1 \mathrm{ml}$ of the culture material $\left(10^{5}\right.$ spirochetes). Mice from the control group were injected at the same time with an equal volume of BSK medium.

2. Infection of mice by infected tick-bite (Figures 1B,C). The nymphs and females that were used in all these experiments were fed on Borrelia-infected mice at the preceding stage. The back of mice was shaved and a hollow plastic cap was glued with wax (Mbow et al., 1994). Ticks were placed in the plastic 
cap that is closed by a plaster. They were observed twice a day.

- Tropism of the various Borrelia strains inoculated by tick bites (comparison with needle inoculation of the various strains). Three females were placed in the plastic cap. Five to seven mice were infected up to 5 days [120 h] of female tick attachment.

- Kinetics of infection of tick salivary glands and of transmission of the different strains after infected tick bite (Figures 1D,E). It was analyzed using ten nymphs or 2-3 females that were allowed to feed on 3 healthy mice for each time of the kinetics. Experiments were repeated 2 or 3 times. The duration of infection was controlled by removing ticks at 12,24 , and $36 \mathrm{~h}$ of feeding for nymphs, and at 24, 72, 120 and repletion for adults. The presence of spirochetes in tick salivary glands and in tick organs was analyzed by PCR.

3. Detection of Borrelia in mice infected by inoculation or tick bite (Figures 1A,E). To determine Borrelia infection in mice, ear biopsies were collected each 7 days after 2 weeks of infection until Borrelia DNA detection. After 38 days of B. burgdorferi sl inoculation or tick bite infection (the timing started as soon as the tick was attached), mice were dissected and spirochete DNA presence was investigated from different organs (brain, heart, back skin, ankle, bladder, muscle, spleen, liver, mesentery and inguinal lymph nodes), collected aseptically.

\section{Dissections}

Tick dissections were performed under microscope in sterile PBS. All dissection materials were cleaned with alcohol $70 \%$ and rinsed with sterile water between each sample. Individual pairs of tick salivary glands, the remaining tick carcasses and all organs from mice were analyzed immediately or frozen at $-80^{\circ} \mathrm{C}$ until DNA extraction.

\section{B. burgdorferi sI DNA Detection in Ticks and Mice}

DNA extraction from ticks or mice was performed using the DNeasy Blood and Tissue Kit (Qiagen, Hilden, Germany). The purified DNA was eluted with sterile water in $20 \mu \mathrm{l}$ for salivary gland, $50 \mu \mathrm{l}$ for nymph carcasses, $100 \mu \mathrm{l}$ for adult carcasses and between 100 and $200 \mu l$ for each mouse organ.

A nested PCR was performed on each sample to amplify the variable spacer region between two tandemly duplicated genes encoding for ribosomal 23 and 5S. The first set of primers used was Ins1 (5'-GAAAAGAGGAAACACCTGTT-3') and S23R (5' TCGGTAATCTTGGGATCAAT-3') which amplified a 360-bp fragment. The second amplification was performed with RRC ( $5^{\prime}-$ CTGCGAGTTCGCGGGAGAG-3') and RRB (5'-AAGCTCCTA GGCATTCACCATA-3') primers resulting in a 257-bp fragment (Schwartz et al., 1992).

For each PCR, $5 \mu \mathrm{l}$ of DNA from the extract or from the first amplification were used. The PCR cycles were carried out with an initial denaturation step for $4 \mathrm{~min}$ at $94^{\circ} \mathrm{C}$; 35 cycles of denaturation for $1 \mathrm{~min}$ at $94^{\circ} \mathrm{C}$, annealing for $1 \mathrm{~min}$ at $55^{\circ} \mathrm{C}$ for
Ins1-S23R and $59^{\circ} \mathrm{C}$ for RRB'-RRC', and extension for $1 \mathrm{~min}$ at $72^{\circ} \mathrm{C}$; and a final extension step at $72^{\circ} \mathrm{C}$ for $10 \mathrm{~min}$.

Each reaction was conducted in a total volume of $25 \mu \mathrm{l}$ with $0.3 \mu \mathrm{mol} / \mu \mathrm{l}$ of each primer, $200 \mu \mathrm{mol} / \mathrm{L}$ of each dNTP, $2.5 \mu \mathrm{l}$ of $10 \times$ PCR buffer, and $1.25 \mathrm{U}$ of Taq DNA polymerase (Taq CORE Kits, Q-biogene). All PCRs were performed in a thermocycler My cycler (Bio-Rad, France). Positive control (B. burgdorferi sl strain different from the strain used for infection) and negative control (PCR mix) were used in each PCR.

PCR products were electrophoresed in a $2 \%$ agarose gel with ethidium bromide and visualized with a Gel Doc ${ }^{\mathrm{TM}}$ XR (BioRad).

\section{Statistical Analysis}

All statistical analyses were done in Simstat (Provalis Research) using the non-parametric tests Kruskal-Wallis and MannWhitney, taking into account the Bonferroni correction.

\section{RESULTS}

\section{Infection of Mice After B. burgdorferi sI Needle Inoculation}

All DNA extraction from ear biopsies of mice infected by $B$. burgdorferi ss B31 strain allowed identification of B. burgdorferi sl DNA after 21 days of infection. For B. burgdorferi ss BRE-13 strain, Borrelia DNA was detected in ear biopsies from $20 \%$ of mice after 14 days and $70 \%$ of mice after 21 days. $90 \%$ of mice were found positive by specific DNA amplification 14 days after inoculation of the B. burgdorferi ss N40 strain. DNA $B$. burgdorferi sl was detected in the ears of mice infected with B. bavariensis $\mathrm{PBi}$ strain after 3 weeks in $60 \%$ of them and in $80 \%$ of mice after 28 days. Borrelia DNA was amplified in $40 \%$ of mice 2 weeks after B. afzelii IBS- 5 inoculation and $80 \%$ of them were positive 1 week later. Thirty eight days after needle inoculation, all mice were found positive for all Borrelia strains.

For the 5 strains, ten organs were studied in order to detect B. burgdorferi sl infection by nested PCR (Table 2, Figure 2). If we consider the three strains of B. burgdorferi ss, B31, BRE13 and N40, specific bacterial amplification was obtained from bladder, back skin and ankle. No B. burgdorferi sl DNA was detected in brain 38 days after inoculation of B31 and N40 strains. BRE-13 strain was the only one for which DNA amplification was observed in the brain while B31 was the only strain of B. burgdorferi ss to be found in the heart.

After inoculation with $B$. bavariensis $\mathrm{PBi}$ strain, bacterial DNA amplification was obtained from bladders, brains and hearts. No bacterial DNA was amplified from back skins and ankles.

Specific DNA was amplified after B. afzelii IBS-5 inoculation from hearts, brains, back skins, ankles and bladders.

We then compared the tropism of the various B. burgdorferi sl strains after injection. Difference of prevalence was observed in various organs: brain, heart, back skin and ankle. As described above, the three $B$. burgdorferi ss strains did not give similar results. B31 and N40 were never present in the brain at the difference of BRE-13 and PBi $(p=0.0003)$. BRE-13 and N40 were not identified in the heart at the difference of PBi $(p=0.0002)$. B31 and N40 were more prevalent in the ankle than PBi 


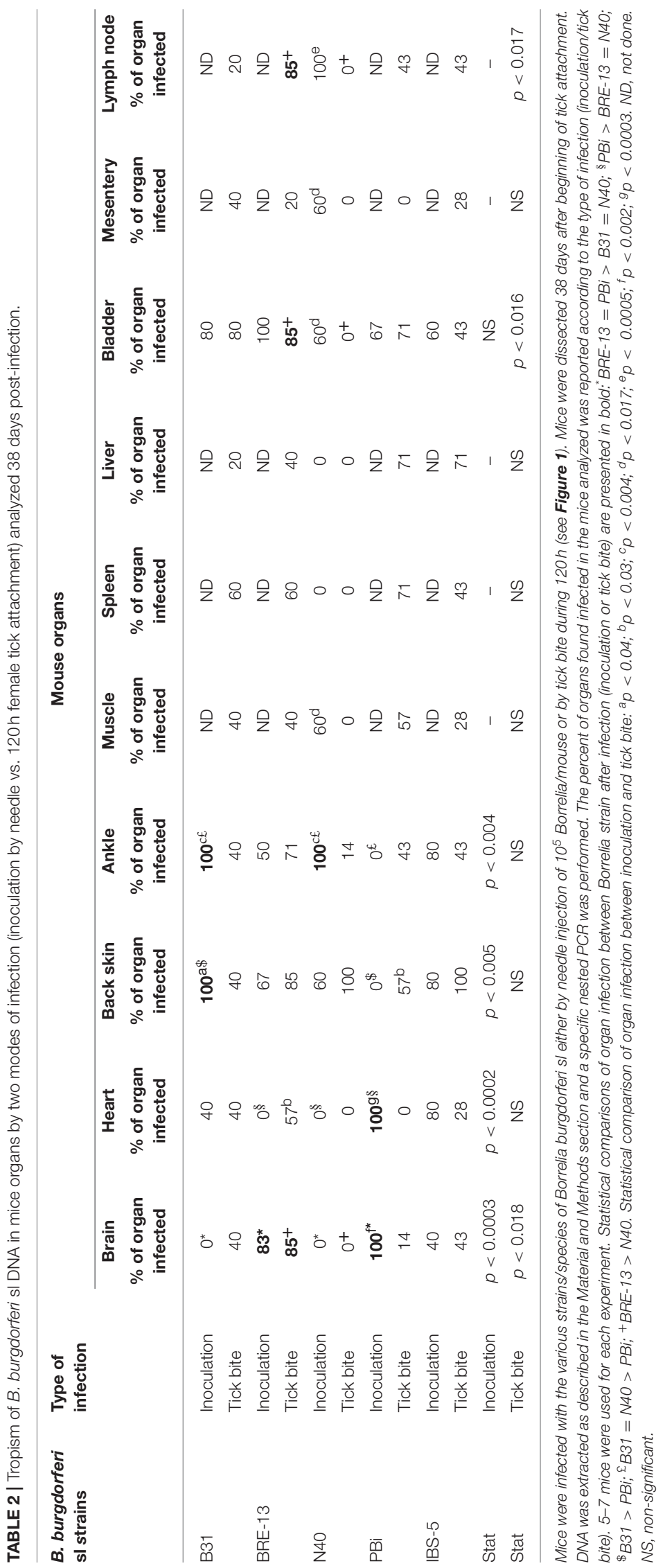



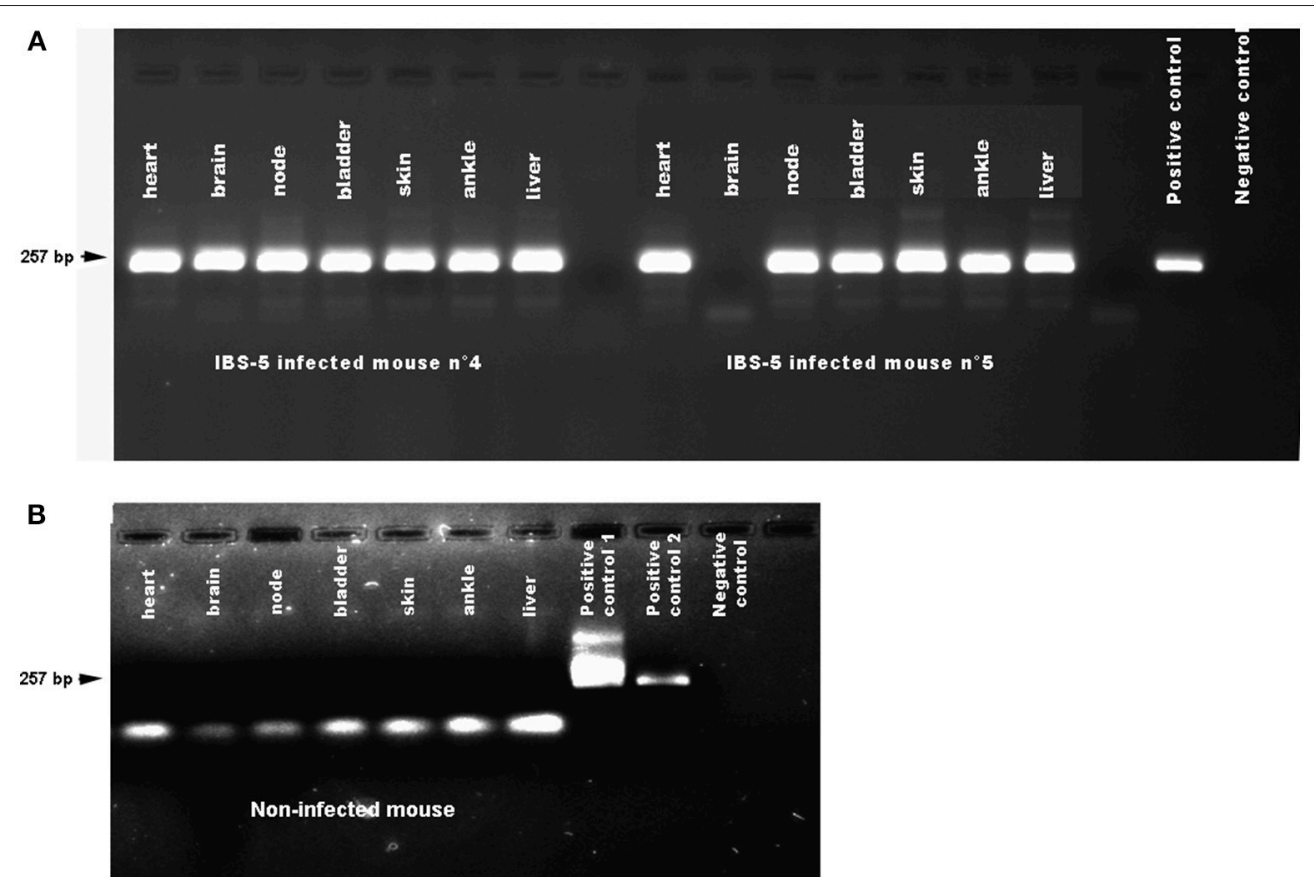

FIGURE 2 | Detection of Borrelia afzelii IBS-5 by nested PCR in the organs of mice after needle inoculation. Mice were infected by subcutaneous inoculation of $10^{5}$ bacteria. Organs were dissected at 38 days post-infection. DNA was extracted as described in the Methods. A first step of PCR was performed followed by a second step of nested PCR. Negative controls (PCR mix) and positive controls (DNA extracted from a culture of Borrelia burgdorferi sl bacteria) were used on each gel. (A) Organs of mice infected by B. afzelii IBS-5. The positive control was a DNA extracted from B. garinii 20047 strain. (B) Organs of a non-infected mouse. Two positive controls were used. Positive control n`1: B. garinii 20047 strain amplified in the first PCR. Positive control 2: B. garinii 20047 strain; Negative control: PCR mix.

$(p<0.004)$. B31 was found more prevalent than PBi in the back skin $(p<0.005)$. IBS-5 was identified in all examined organs while PBi was not found in the back skin and ankle.

\section{B. burgdorferi sl Infection in I. ricinus Ticks After Infection on Needle-Infected Mice}

A total of 2,400 nymphs and about 1,500 larvae were used to obtain infected ticks.

On 400 nymphs used for each strain, 224 (56\%), 284 (71\%), $315(79 \%), 233(58 \%)$, and $270(68 \%)$ were engorged and spontaneously detached from mice infected respectively with B. burgdorferi ss B31, BRE-13, and N40, B. bavariensis PBi and B. afzelii IBS-5 strains. After 3 months, respectively 145 (65\%), 247 (87\%), 255 (81\%), 116 (50\%), and 261 (97\%) nymphs molted into adults from which 79, 93, 120, 45, and 109 females were obtained for each strain.

Female infection rates of the 3 B. burgdorferi ss strains reached $70 \%$ for B31 (16/23), 78\% (28/36) for N40 and 96\% for BRE13 (27/28), the latter strain having the highest rate of infection of the $B$. burgdorferi ss strains $(p=0.008)$. Infection rates in females were $70 \%$ (23/33) for B. bavariensis PBi and 70\% (21/30) for B. afzelii IBS-5. When all strains were compared, their rate of infection was similar.

Concerning larvae, 338, 360, and 390 of them were engorged from mice infected respectively with $B$. burgdorferi ss BRE-13, B. bavariensis $\mathrm{PBi}$ and $B$. afzelii IBS-5 strains. After 3 months, respectively 59,60 , and $61 \%$ of $\mathrm{BRE}-13, B$. bavariensis $\mathrm{PBi}$ and $B$. afzelii IBS-5 engorged larvae molted into nymphs. The infection rates in nymphs were $90 \%$ (27/30) for B. burgdorferi ss BRE-13, 70\% (21/30) for B. bavariensis PBi and 77\% (23/30) for B. afzelii IBS-5.

\section{B. burgdorferi sl Tropism in Mice After Infected Tick Bite: Comparison With Needle Injection}

Mice were infected by ticks that were removed at $120 \mathrm{~h}$ of tick attachment. They were sacrificed at 38 days post-infection and their organs dissected. Borrelia DNA was detected after nested PCR. The percent of positive organs as function of the strain is reported in Table 2 .

After tick bite infection with B. burgdorferi ss B31 and BRE13 strain, all organs presented bacterial DNA amplification with various infection rates while in $\mathrm{N} 40$-infected mice, only ankles and back skin were found infected. After bite of B. bavariensis PBi infected ticks, bacterial DNA amplification was obtained from all organs except heart and mesentery. B. burgdorferi DNA was amplified after bite of $B$. afzelii IBS-5 infected females from all tested organs.

When the prevalence of all strains were compared in each organ, statistical analyses showed that B. burgdorferi ss BRE13 was more prevalent than $B$. burgdorferi ss N40 in the brain $(p<0.018)$, in the bladder $(p<0.016)$ and in the inguinal lymph node $(p<0.017)$ after tick bite. 
We compared the dissemination of each strain in the various organs after inoculation or tick bite. We observed the infection of the heart after tick inoculation of B. burgdorferi ss BRE-13 whereas this organ was found uninfected after inoculation. The same trend was also observed for B. bavariensis PBi for which an infection of the back skin was only detected after tick bite. For the other comparisons, more organs were found infected after inoculation than after tick bite. For instance, a higher number of positive organs was statistically found after inoculation of B. burgdorferi ss N40 compared with tick bite.

\section{Adult Tick Infection Kinetics of Salivary Glands and Mice by B. Burgdorferi sl After Female Tick Bite}

All results of B. burgdorferi sl DNA detection in female salivary glands and corresponding mice are reported in Table 3. Mouse infection was detected 38 days after removing infected female ticks.

Concerning B. burgdorferi ss B31 infected ticks, salivary gland extract DNA showed no Borrelia amplification for tick attachment of $24 \mathrm{~h}$. But after $120 \mathrm{~h}$ of female feeding, the specific DNA fragment was amplified from $67 \%$ of the salivary glands tested. $100 \%$ of the mice were found infected for tick attachment of $120 \mathrm{~h}$.

The B. burgdorferi ss N40 strain was detected in tick salivary glands collected at $24 \mathrm{~h}$ of tick fixation with bacterial DNA amplification from $50 \%$ of female salivary glands. At respectively 72 and $120 \mathrm{~h}$ of tick attachment, 100 and $83 \%$ of salivary glands showed B. burgdorferi sl DNA amplification. Furthermore, 100\% of mice became infected by N40 strain up to $24 \mathrm{~h}$ of tick attachment as well as up to 72 and $120 \mathrm{~h}$ of tick bite.

Concerning the 3 other strains, B. burgdorferi ss BRE-13, B. bavariensis $\mathrm{PBi}$ and $B$. afzelii IBS-5, B. burgdorferi sl DNA was amplified from $100 \%$ of female salivary glands infected at the preceding stage prior to their fixation on mice. From $24 \mathrm{~h}$ to repletion, the rate of infection of tick salivary glands with the three different strains was shown to vary depending on the time (Table 3).

When ticks were removed at $24 \mathrm{~h}$ of tick attachment, $B$. burgdorferi sl DNA was detected in mice for the three strains: $100 \%$ was infected by B. burgdorferi ss BRE-13, B. bavariensis $\mathrm{PBi}$ and $B$. afzelii IBS-5. This rate of infection was constant after bite of $B$. burgdorferi ss BRE-13 and B. afzelii IBS- 5 whatever tick attachment time, whereas a slight decrease was observed at $120 \mathrm{~h}$ of tick attachment for B. bavariensis PBi (Table 3).

We then tested the tropism of the various strains as function of the time of attachment of infected ticks (Table 4). Again, significant differences were found between the various strains of B. burgdorferi ss. BRE-13 was the one for which all organs tested except the heart was found infected after $24 \mathrm{~h}$ of tick attachment, whereas only the skin and ankle were found infected by N40 and none by B31. Ankles were found positive for all strains except B31 while only BRE-13 and PBi were found in the bladder and in the lymph nodes. The tropism of IBS- 5 and N40 was similar.

After removing the ticks at $72 \mathrm{~h}, \mathrm{PBi}$ was found in all organs except the lymph node and the heart, this latter was never infected by this strain whatever the time of attachment. PBi was found in the brain at $72 \mathrm{~h}$ of tick attachment and was still detected at $120 \mathrm{~h}$ but not any more after repletion of the tick. The bladder was found positive for all strains except N40 after removing the ticks at $120 \mathrm{~h}$ or after repletion. Interestingly, we found fewer organs infected after repletion of the tick whatever the strain.

\section{B. burgdorferi sl Infection Kinetics After Nymph Bites}

No B. burgdorferi sl DNA was amplified from nymph salivary glands infected at the preceding stage prior to their fixation on mice. Mice were found infected as soon as $12 \mathrm{~h}$ of attachment of $B$. burgdorferi ss BRE-13, B. bavariensis $\mathrm{PBi}$ and B. afzelii IBS-5 infected nymphs (Table 5). All organs except lymph node

TABLE 3 | Adult tick infection kinetics of salivary glands and mice by B. burgdorferi sl after female tick bite.

\begin{tabular}{|c|c|c|c|c|c|c|}
\hline \multirow[t]{2}{*}{ B. burgdorferi sl strains } & \multirow[t]{2}{*}{ Samples } & \multicolumn{5}{|c|}{ Infection rate $\%$} \\
\hline & & Unfed ticks & $24 h$ & $72 \mathrm{~h}$ & $120 \mathrm{~h}$ & Repletion \\
\hline \multirow[t]{2}{*}{ B31 } & Tick salivary glands N (\%) & $0 / 3(0)^{\star}$ & $0 / 6(0)^{\$}$ & ND & $4 / 6(67)$ & ND \\
\hline & Mice (\%) & - & 0 & ND & 100 & ND \\
\hline \multirow[t]{2}{*}{ BRE-13 } & Tick salivary glands N (\%) & $3 / 3(100)^{\star}$ & $3 / 3(100)^{\$}$ & $6 / 6(100)$ & $4 / 4(100)$ & $5 / 5(100)$ \\
\hline & Mice (\%) & - & 100 & 100 & 100 & 100 \\
\hline \multirow[t]{2}{*}{ N40 } & Tick salivary glands N (\%) & $0 / 3(0)^{\star}$ & $2 / 4(50)$ & 6/6 (100) & 5/6 (83) & $4 / 4(100)$ \\
\hline & Mice (\%) & - & 100 & 100 & 100 & 100 \\
\hline \multirow[t]{2}{*}{ PBi } & Tick salivary glands N (\%) & $3 / 3(100)^{\star}$ & $3 / 5(60)$ & $3 / 6(50)$ & $6 / 6(100)$ & ND \\
\hline & Mice (\%) & - & 100 & 100 & 86 & 100 \\
\hline \multirow[t]{2}{*}{ IBS-5 } & Tick salivary glands N (\%) & $3 / 3(100)^{\star}$ & $2 / 5(40)$ & $3 / 5(50)$ & $2 / 4(50)$ & $4 / 4(100)$ \\
\hline & Mice (\%) & - & 100 & 100 & 100 & 100 \\
\hline Stat & Tick salivary glands & $p<0.003$ & $p<0.04$ & NS & NS & NS \\
\hline
\end{tabular}

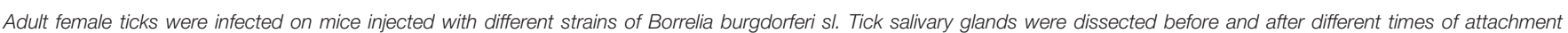

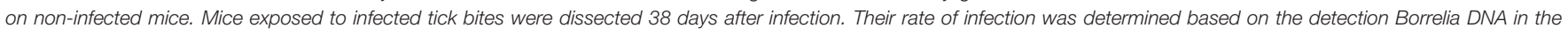

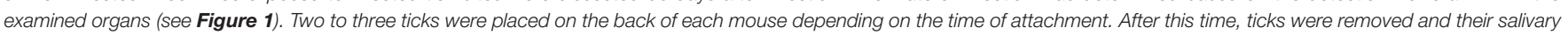

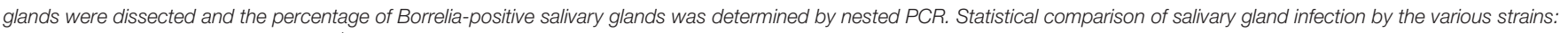
${ }^{*} B R E-13=P B i=I B S 5>B 31=N 40 ;{ }^{\$} B R E-13>B 31 . N D$, not done; NS, non-significant. 
TABLE 4 | Tropism of B. burgdorferi sl strains in mice after various durations of female tick attachment.

\begin{tabular}{|c|c|c|c|c|c|c|c|c|c|}
\hline & BRE-13 & PBi & IBS-5 & N40 & B31 & BRE-13 & $\mathbf{P B i}$ & IBS-5 & N40 \\
\hline & \multicolumn{5}{|c|}{$24 \mathrm{~h}$} & \multicolumn{4}{|c|}{$72 \mathrm{~h}$} \\
\hline Brain & $1 / 1$ & $0 / 1$ & $0 / 1$ & $0 / 1$ & $0 / 2$ & $1 / 1$ & $1 / 1$ & $0 / 1$ & $0 / 1$ \\
\hline Heart & $0 / 1$ & $0 / 1$ & $0 / 1$ & $0 / 1$ & $0 / 2$ & $1 / 1$ & $0 / 1$ & $0 / 1$ & $0 / 1$ \\
\hline Skin & $1 / 1$ & $0 / 1$ & $1 / 1$ & $1 / 1$ & $0 / 2$ & $0 / 1$ & $1 / 1$ & $1 / 1$ & $1 / 1$ \\
\hline Ankle & $1 / 1$ & $1 / 1$ & $1 / 1$ & $1 / 1$ & $0 / 2$ & $1 / 1$ & $1 / 1$ & $1 / 1$ & $0 / 1$ \\
\hline Bladder & $1 / 1$ & $1 / 1$ & $0 / 1$ & $0 / 1$ & $0 / 2$ & $1 / 1$ & $1 / 1$ & $0 / 1$ & $0 / 1$ \\
\hline \multirow[t]{2}{*}{ Lymph node } & $1 / 1$ & $1 / 1$ & $0 / 1$ & $0 / 1$ & $0 / 2$ & $1 / 1$ & $0 / 1$ & $1 / 1$ & $0 / 1$ \\
\hline & \multicolumn{5}{|c|}{$120 h$} & \multicolumn{4}{|c|}{ Repletion } \\
\hline Brain & $6 / 7$ & $1 / 7$ & $3 / 7$ & $0 / 7$ & $2 / 5$ & $1 / 1$ & $0 / 1$ & $0 / 1$ & $0 / 1$ \\
\hline Heart & $4 / 7$ & $0 / 7$ & $2 / 7$ & $0 / 7$ & $2 / 5$ & $0 / 1$ & $0 / 1$ & $1 / 1$ & $0 / 1$ \\
\hline Skin & $6 / 7$ & $4 / 7$ & $7 / 7$ & $7 / 7$ & $2 / 5$ & $0 / 1$ & $1 / 1$ & $1 / 1$ & $1 / 1$ \\
\hline Ankle & $5 / 7$ & $3 / 7$ & $3 / 7$ & $1 / 7$ & $4 / 5$ & $1 / 1$ & $1 / 1$ & $0 / 1$ & $1 / 1$ \\
\hline Bladder & $6 / 7$ & $5 / 7$ & $3 / 7$ & $0 / 7$ & $4 / 5$ & $1 / 1$ & $1 / 1$ & $1 / 1$ & $0 / 1$ \\
\hline Lymph node & $6 / 7$ & $3 / 7$ & $3 / 7$ & $0 / 7$ & $1 / 5$ & $1 / 1$ & $0 / 1$ & $1 / 1$ & $0 / 1$ \\
\hline
\end{tabular}

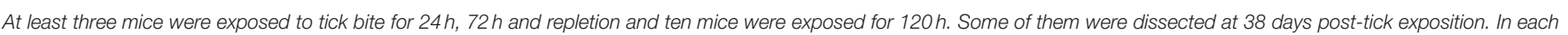
column are presented the number of mice infected (based on the detection of Borrelia DNA by nested PCR in the organs) divided by the number tested.

were found positive after removing nymphs at $12 \mathrm{~h}$ for BRE-13 whereas only the ankle and lymph node were infected for PBi, and the brain for IBS-5 after the same time of contact with the infected nymphs.

\section{DISCUSSION}

Borrelia burgdorferi sl is transmitted to the host during Ixodes ticks blood feeding. Several studies have shown the role that tick saliva could play in tick feeding and pathogen transmission. The minimal infected tick attachment time on a vertebrate host for efficient transmission is still a matter of debate (Cook, 2015). Indeed, it is frequently specified that the risk of infection is lower if the tick is removed within 24-48 h (Piesman et al., 1987; des Vignes et al., 2001). An increased risk of infection is observed with longer tick attachment durations. Several studies showed that a blood meal is able to trigger the downregulation of OspA/B (involved in the colonization and survival of the tick midgut) and the upregulation of OspC (involved in the dissemination from the midgut to the salivary glands in the tick, and the interaction with the vertebrate host) (Grimm et al., 2004; Stewart et al., 2006; Fingerle et al., 2007; Kenedy et al., 2012). These processes are suggested to prepare the bacteria for the infection of vertebrate hosts.

However, data from the literature showed that transmission can occur in less than $16 \mathrm{~h}$ (Hynote et al., 2012). In most unfed ticks, spirochetes were shown to be present in the midgut and need to migrate during blood feeding to the salivary glands, from which they are transmitted to the host via saliva (Ribeiro et al., 1987; Zung et al., 1989). However, disseminated infection was observed in ticks before feeding (Piesman et al., 1987). Moreover, there is evidence that Borrelia transmission and virulence depend on tick and Borrelia species (Moskvitina et al., 1995a,b).

In the present study, the efficiency and the kinetics at which Ixodes ricinus ticks are able to transmit $B$. afzelii, B. bavariensis and several strains of $B$. burgdorferi ss to mice were examined in relation to blood meal length. We also examined the tropism of these various strains according to the transmission mode: infected tick bite vs. needle inoculation.

B31 and N40 are two widely studied strains of B. burgdorferi ss, which belong to two different $16 \mathrm{~S}-23 \mathrm{~S}$ rRNA spacer types and outer surface protein C (OspC) allelic groups. They were both isolated from Ixodes scapularis ticks, and both are very infectious in the mouse model (Burgdorfer et al., 1982; Barthold et al., 1988). Although phylogenetic analyses predicted B31 to be more infectious, Chan et al. (Chan et al., 2012) indicated that N40 strain was more infectious at lower doses of inoculation. BRE-13 belongs to another group of OspC and was isolated from the CSF of a patient with neurological symptoms (B. Degeilh, personal communication).

Our observations showed that the two B. burgdorferi ss N40 and B31 strains were not present in unfed adult tick salivary glands and that the transmission to mice was observed earlier (24h) for N40 and from $120 \mathrm{~h}$ after tick attachment for B31. This was not what we observed for BRE-13, another strain of $B$. burdorferi ss, for which unfed adult ticks had systemic infections. We thus observed a strain-specific variation in the tissue tropism in ticks. However, since we set the first infection duration at $24 \mathrm{~h}$, we were not able to see any difference in the kinetics of infection in mouse between BRE-13 and N40.

B. afzelii IBS-5 and B. bavariensis PBi were also present in unfed adult tick salivary glands, as was previously shown for nymphs infected by B. garinii and B. afzelii (Kahl et al., 1998). In agreement with this observation, mice were found infected as early as $24 \mathrm{~h}$ of tick attachment.

For ticks with systemic infection, we did not find that the risk of transmission increased with the contact duration with host since all mice were found infected whatever the duration of attachment. For strains like B31 and N40 that were not found in salivary glands before feeding, we found 
TABLE 5 | Kinetics and tropism of Borrelia transmission to mice by infected nymphs.

\begin{tabular}{|c|c|c|c|c|c|c|c|c|c|c|c|}
\hline Borrelia strain & $\begin{array}{c}\text { Time of tick } \\
\text { attachment (h) }\end{array}$ & Brain & Heart & Back skin & Ankle & Muscle & Spleen & Liver & Bladder & Mesentery & Lymph node \\
\hline \multirow[t]{3}{*}{ BRE-13 } & 12 & + & + & + & + & + & + & + & + & + & - \\
\hline & 24 & + & + & - & - & + & + & - & - & - & + \\
\hline & 36 & + & - & + & + & + & + & + & + & - & - \\
\hline $\mathrm{PBi}$ & 12 & - & - & - & + & - & - & - & - & - & + \\
\hline IBS-5 & 12 & + & - & - & - & - & - & - & - & - & - \\
\hline
\end{tabular}

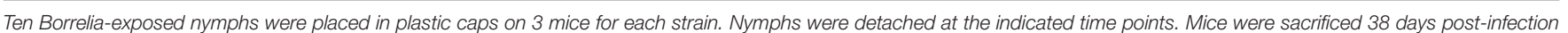

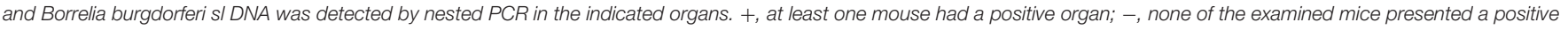
organ.

a difference in the kinetics of infection. B31 has not reached the salivary glands at $24 \mathrm{~h}$ after tick attachment while N40 was certainly present in this organ before $24 \mathrm{~h}$ and able to infect the mice. We were also able to show that the dissemination of the various strains differed according to the duration of attachment. For instance, BRE-13 was isolated in several organs except the heart after only $24 \mathrm{~h}$ of tick attachment, the heart being found infected after $72 \mathrm{~h}$ of tick feeding. PBi was only identified in the brain when removing the tick after $72 \mathrm{~h}$ but not before. For IBS-5, all organs were found infected only at $120 \mathrm{~h}$ of tick attachment. These observations may suggest that the symptomatology and thereby the severity of the infection may be correlated to the duration of attachment, at least for some strains, since BRE-13 seems virulent shortly after tick attachment and N40 infects the same organs whatever the time of feeding.

We also studied the infected nymph attachment duration to obtain infected mice. We did not find any systemic infection in B. burdorferi ss BRE-13, B. bavariensis PBi and Borrelia afzelii IBS-5 infected nymphs. However, mice were infected when nymphs were attached for less than $12 \mathrm{~h}$. Again almost all organs tested, including the brain and the heart are infected after $12 \mathrm{~h}$ of BRE-13-infected nymph feeding, while PBi was only detected in the ankle and in the lymph nodes and IBS-was identified in the brain.

The ticks, vertebrate hosts and pathogens co-evolution has led to the development of strategies to use salivary components in order to guarantee both pathogen acquisition and transmission, as well as local and disseminated infection in the host (Šimo et al., 2017). Various studies have shown a higher efficiency in Borrelia transmission in presence of saliva or saliva components (Wikel, 2013). The salivary gland protein Salp15 plays an immunosuppressive role and may thereby facilitate infection by the low numbers of spirochetes that are released in the skin during tick feeding (Anguita et al., 2002; Ramamoorthi et al., 2005). A tick antioxidant was shown to facilitate tick acquisition of spirochetes from infected animals (Narasimhan et al., 2007). We were therefore interested in comparing the various $B$. burgdorferi sl strains tropism after needle injection or tick bite. In the murine model, mice can be infected by needle inoculation or tick feeding (Tilly et al., 2008). Most mouse strains, as well as the natural reservoir hosts, display no sign of disease, but develop a serological response to $B$. burgdorferi proteins and a persistent infection (Schwan et al., 1988; Barthold et al., 1990; Wu et al., 2013).

After needle injection, we found a difference of tropism in the brain, in the heart, in the back skin and in the ankle for the different strains tested. B. burdorferi ss BRE-13 and B. bavariensis $\mathrm{PBi}$ were detected in the brain of mice while $B$. burdorferi ss B31 and N40 were never detected. The B. burgdorferi ss strains that we used in this study were shown to present differential tropism. They belong to different OspC groups (Table 1). OspC is known to be involved in the early stages of mouse infection as well as in dissemination (Kenedy et al., 2012). We can therefore hypothesize that this could explain the altered dissemination observed in the brain for B31 and N40 compared to BRE-13. Interestingly, Wu et al. showed that B. burdorferi ss B31 infected mouse brain but it was after intraperitoneal injection of the spirochetes (Wu et al., 2013) suggesting that the intraperitoneal vs. subcutaneous injection route may affect Borrelia bacteria tropism. Moreover, the strain of the mice used in our studies are not the same, Balb/C vs. $\mathrm{C} 3 \mathrm{H} / \mathrm{HeN}$, this latter being the most relevant animal model for Lyme borreliosis for discriminating infectivity and pathogenicity of various $B$. burgdorferi strains (Chan et al., 2012). However, in agreement with Wu et al. (2013) we were also able to show that statistical differences existed between the tropism of B31 and PBi (B. bavariensis but formerly $B$. garinii) in the brain and in the heart.

A significantly higher number of ankles positive for B. burdorferi ss B31, BRE-13 and N40 was observed compared with $B$. bavariensis $\mathrm{PBi}$. These results are in agreement with the association between some clinical manifestations of LB and the species of $B$. burgdorferi sl. Arthritis was shown to be caused prevalently by $B$. burgdorferi ss, and neuroborreliosis by B. burgdorferi ss and B. garinii/B. bavariensis (Balmelli and Piffaretti, 1995).

All organs were found positive after feeding with ticks infected with Borrelia burgdorferi ss B31 and BRE-13 and with Borrelia afzelii IBS-5. Interestingly, all strains except N40 were neurotropic after $120 \mathrm{~h}$ of tick feeding.

When we compared the tropism of the various Borrelia species between tick bite and needle inoculation, it appears that most of the time the percentage of positive organs was higher after inoculation, except for B. burdorferi ss BRE-13 that was found in the heart and for B. bavariensis $\mathrm{PBi}$, which is detected in the back skin only after tick bite. These two examples could illustrate 
the role of tick saliva in Borrelia dissemination and tropism. N40 was the strain for which the highest number of positive organs was observed after needle injection while it was detected in only two organs after tick bite: the back skin and the ankle. These results suggest that the number of Borrelia injected by needle $\left(10^{5}\right)$ may exceed by far what is injected by the tick. When Borrelia bacteria were quantified by Q-PCR in salivary glands of Ixodes ricinus infected by different strains, their level was shown to differ according to the strain (Cotté et al., 2014). The highest level was found in $B$. burdorferi ss BRE-13-infected salivary glands $\left(10^{6}\right)$, while $10^{4}$ bacteria were detected in $B$. burdorferi ss $\mathrm{B} 31$ and $B$. bavariensis $\mathrm{PBi}$ infected glands, the lowest amount, $10^{3}$, being found in $B$. afzelii IBS-5 infected salivary glands.

In conclusion, we showed that salivary glands of adult ticks infected by the strains $B$. burdorferi ss BRE-13, B. burdorferi ss $\mathrm{PBi}$ and $B$. afzelii IBS-5 are infected before feeding. The spirochetes are thus present in the salivary glands, thereby potentially able to be injected immediately after tick attachment to host. One hundred percent of mice were found infected as early as $24 \mathrm{~h}$ of tick attachment. Interestingly, in contradiction with other studies, we did not report any increase in mice infection rate depending on feeding time, while it is observed for B. burdorferi ss B31. We also showed that even though the salivary glands of unfed nymphs infected by $B$. burgdorferi ss BRE-13 B. bavariensis PBi and B. afzelii IBS-5 are not infected, Borrelia transmission was shown to occur as early as $12 \mathrm{~h}$ of

\section{REFERENCES}

Anguita, J., Ramamoorthi, N., Hovius, J. W., Das, S., Thomas, V., Persinski, R., et al. (2002). Salp15, an Ixodes scapularis salivary protein, inhibits CD4 ${ }^{+} \mathrm{T}$ cell activation. Immunity 16, 849-859. doi: 10.1016/S1074-7613(02)00325-4

Balmelli, T., and Piffaretti, J. C. (1995). Association between different clinical manifestations of Lyme disease and different species of Borrelia burgdorferi sensu lato. Res. Microbiol. 146, 329-340.

Barthold, S. W., Beck, D. S., Hansen, G. M., Terwilliger, G. A., and Moody, K. D. (1990). Lyme borreliosis in selected strains and ages of laboratory mice. J. Infect. Dis. 162, 133-138.

Barthold, S. W., Moody, K. D., Terwilliger, G. A., Jacoby, R. O., and Steere, A. C. (1988). An animal model for Lyme arthritis. Ann. N Y Acad. Sci. 539, 264-273.

Burgdorfer, W., Barbour, A. G., Hayes, S. F., Benach, J. L., Grunwaldt, E., and Davis, J. P. (1982). Lyme disease-a tick-borne spirochetosis? Science 216, 1317-1319.

Chan, K., Awan, M., Barthold, S. W., and Parveen, N. (2012). Comparative molecular analyses of Borrelia burgdorferi sensu stricto strains B31 and N40D10/E9 and determination of their pathogenicity. BMC Microbiol. 12:157. doi: 10.1186/1471-2180-12-157

Coipan, E. C., Jahfari, S., Fonville, M., Oei, G. A., Spanjaard, L., Takumi, K., et al. (2016). Imbalanced presence of Borrelia burgdorferi s.l. multilocus sequence types in clinical manifestations of Lyme borreliosis. Infect. Genet. Evol. 42, 66-76. doi: 10.1016/j.meegid.2016.04.019

Collares-Pereira, M., Couceiro, S., Franca, I., Kurtenbach, K., Schafer, S. M., Vitorino, L., et al. (2004). First isolation of Borrelia lusitaniae from a human patient. J. Clin. Microbiol. 42, 1316-1318. doi: 10.1128/JCM.42.3.1316-1318.2004

Cook, M. J. (2015). Lyme borreliosis: a review of data on transmission time after tick attachment. Int. J. Gen. Med. 8, 1-8. doi: 10.2147/IJGM.S73791

Cotté, V., Sabatier, L., Schnell, G., Carmi-Leroy, A., Rousselle, J. C., Arsene-Ploetze, F., et al. (2014). Differential expression of Ixodes ricinus salivary gland proteins tick attachment. It is therefore important to remove nymphs or adult ticks as soon as possible after bite to prevent any transmission.

Borrelia bacteria tropism tested in this study was shown to vary according to the strain as well as between ticks bite and needle inoculation. These results confirm the association between some strains and clinical manifestation of the LB, as well as the role played by tick saliva in the efficiency of Borrelia bacterial infection and dissemination in vertebrates.

\section{AUTHOR CONTRIBUTIONS}

$\mathrm{VCH}$ and EF designed and analyzed the experiments. NS, VCO, and MG performed and analyzed the experiments. LM provided the ticks for the experiments. VCH, EF, and LM wrote the manuscript.

\section{FUNDING}

This work was funded by the PTR 309 (Institut Pasteur) and by the Institut de Veille Sanitaire.

\section{ACKNOWLEDGMENTS}

We thank our colleagues B. Degeilh, B. Jaulhac, C.S. Pavia, and G. Chaconas for providing us with some strains that we used in this work. in the presence of the Borrelia burgdorferi sensu lato complex. J. Proteomics 96, 29-43. doi: 10.1016/j.jprot.2013.10.033

des Vignes, F., Piesman, J., Heffernan, R., Schulze, T. L., Stafford, K. C. III, and Fish, D. (2001). Effect of tick removal on transmission of Borrelia burgdorferi and Ehrlichia phagocytophila by Ixodes scapularis nymphs. J. Infect. Dis. 183, 773-778. doi: 10.1086/318818

Eisen, L. (2018). Pathogen transmission in relation to duration of attachment by Ixodes scapularis ticks. Ticks Tick Borne Dis. 9, 535-542. doi: 10.1016/j.ttbdis.2018.01.002

Fingerle, V., Goettner, G., Gern, L., Wilske, B., and Schulte-Spechtel, U. (2007). Complementation of a Borrelia afzelii OspC mutant highlights the crucial role of OspC for dissemination of Borrelia afzelii in Ixodes ricinus. Int. J. Med. Microbiol. 297, 97-107. doi: 10.1016/j.ijmm.2006.11.003

Fraser, C. M., Casjens, S., Huang, W. M., Sutton, G. G., Clayton, R., Lathigra, R., et al. (1997). Genomic sequence of a Lyme disease spirochaete, Borrelia burgdorferi. Nature 390, 580-586. doi: 10.1038/37551

Gern, L., Schaible, U. E., and Simon, M. M. (1993). Mode of inoculation of the Lyme disease agent Borrelia burgdorferi influences infection and immune responses in inbred strains of mice. J. Infect. Dis. 167, 971-975.

Grimm, D., Tilly, K., Byram, R., Stewart, P. E., Krum, J. G., Bueschel, D. M., et al. (2004). Outer-surface protein C of the Lyme disease spirochete: a protein induced in ticks for infection of mammals. Proc. Natl. Acad. Sci. U.S.A. 101, 3142-3147. doi: 10.1073/pnas.0306845101

Hynote, E. D., Mervine, P. C., and Stricker, R. B. (2012). Clinical evidence for rapid transmission of Lyme disease following a tickbite. Diagn. Microbiol. Infect. Dis. 72, 188-192. doi: 10.1016/j.diagmicrobio.2011.10.003

Kahl, O., Janetzki-Mittmann, C., Gray, J. S., Jonas, R., Stein, J., and de Boer, R. (1998). Risk of infection with Borrelia burgdorferi sensu lato for a host in relation to the duration of nymphal Ixodes ricinus feeding and the method of tick removal. Zentralbl. Bakteriol. 287, 41-52. 
Kenedy, M. R., Lenhart, T. R., and Akins, D. R. (2012). The role of Borrelia burgdorferi outer surface proteins. FEMS Immunol. Med. Microbiol. 66, 1-19. doi: 10.1111/j.1574-695X.2012.00980.x

Koedel, U., Fingerle, V., and Pfister, H. W. (2015). Lyme neuroborreliosisepidemiology, diagnosis and management. Nat. Rev. Neurol. 11, 446-456. doi: $10.1038 /$ nrneurol.2015.121

Labuda, M., and Nuttall, P. A. (2004). Tick-borne viruses. Parasitology 129, S221-S245. doi: 10.1017/S0031182004005220

Lagal, V., Postic, D., Ruzic-Sabljic, E., and Baranton, G. (2003). Genetic diversity among Borrelia strains determined by single-strand conformation polymorphism analysis of the ospC gene and its association with invasiveness. J. Clin. Microbiol. 41, 5059-5065. doi: 10.1128/jcm.41.11.5059-5065.2003

Lindgren, E., and Jaenson, T. G. (2006). Lyme Borreliosis in Europe: Influences of Climate and Climate Change, Epidemiology, Ecology and Adaptation Measures. Copenhagen: WHO Regional Office for Europe.

Margos, G., Vollmer, S. A., Cornet, M., Garnier, M., Fingerle, V., Wilske, B., et al. (2009). A new Borrelia species defined by multilocus sequence analysis of housekeeping genes. Appl. Environ. Microbiol. 75, 5410-5416. doi: 10.1128/aem.00116-09

Mbow, M. L., Christe, M., Rutti, B., and Brossard, M. (1994). Absence of acquired resistance to nymphal Ixodes ricinus ticks in BALB/c mice developing cutaneous reactions. J. Parasitol. 80, 81-87.

Moskvitina, G. G., Korenberg, E. I., and Gorban, L. (1995a). [The presence of Borrelia in the intestines and salivary glands of spontaneously infected adult Ixodes persulcatus Schulze ticks during bloodsucking]. Med. Parazitol. 3, 16-20.

Moskvitina, G. G., Korenberg, E. I., Spielman, A., and Shchegoleva, T. V. (1995b). [The frequency of generalized infection in adult fasting ticks of the genus Ixodes in foci of borreliosis in Russia and the USA]. Parazitologiia 29, 353-360.

Narasimhan, S., Sukumaran, B., Bozdogan, U., Thomas, V., Liang, X., DePonte, K., et al. (2007). A tick antioxidant facilitates the Lyme disease agent's successful migration from the mammalian host to the arthropod vector. Cell. Host. Microbe 2, 7-18. doi: 10.1016/j.chom.2007.06.001

Pachner, A. R., and Itano, A. (1990). Borrelia burgdorferi infection of the brain: characterization of the organism and response to antibiotics and immune sera in the mouse model. Neurology 40, 1535-1540.

Piesman, J., Mather, T. N., Sinsky, R. J., and Spielman, A. (1987). Duration of tick attachment and Borrelia burgdorferi transmission. J. Clin. Microbiol. 25, 557-558.

Ramamoorthi, N., Narasimhan, S., Pal, U., Bao, F., Yang, X. F., Fish, D., et al. (2005). The Lyme disease agent exploits a tick protein to infect the mammalian host. Nature 436, 573-577. doi: 10.1038/nature03812

Ribeiro, J. M., Mather, T. N., Piesman, J., and Spielman, A. (1987). Dissemination and salivary delivery of Lyme disease spirochetes in vector ticks (Acari: Ixodidae). J. Med. Entomol. 24, 201-205.

Rijpkema, S. G., Tazelaar, D. J., Molkenboer, M. J., Noordhoek, G. T., Plantinga, G., Schouls, L. M., et al. (1997). Detection of Borrelia afzelii, Borrelia burgdorferi sensu stricto, Borrelia garinii and group VS116 by PCR in skin biopsies of patients with erythema migrans and acrodermatitis chronica atrophicans. Clin. Microbiol. Infect. 3, 109-116.

Rudenko, N., Golovchenko, M., Grubhoffer, L., and Oliver, J. H. Jr. (2011). Updates on Borrelia burgdorferi sensu lato complex with respect to public health. Ticks Tick Borne Dis. 2, 123-128. doi: 10.1016/j.ttbdis.2011. 04.002
Schwan, T. G., Burgdorfer, W., Schrumpf, M. E., and Karstens, R. H. (1988). The urinary bladder, a consistent source of Borrelia burgdorferi in experimentally infected white-footed mice (Peromyscus leucopus). J. Clin. Microbiol. 26, 893-895.

Schwartz, I., Wormser, G. P., Schwartz, J. J., Cooper, D., Weissensee, P., Gazumyan, A., et al. (1992). Diagnosis of early Lyme disease by polymerase chain reaction amplification and culture of skin biopsies from erythema migrans lesions. $J$. Clin. Microbiol. 30, 3082-3088.

Šimo, L., Kazimirova, M., Richardson, J., and Bonnet, S. I. (2017). The Essential Role of Tick Salivary Glands and Saliva in Tick Feeding and Pathogen Transmission. Front. Cell. Infect. Microbiol. 7:281. doi: 10.3389/fcimb.2017.00281

Skotarczak, B. (2009). Adaptation factors of Borrelia for host and vector. Ann. Agric. Environ. Med. 16, 1-8.

Stanek, G., Fingerle, V., Hunfeld, K. P., Jaulhac, B., Kaiser, R., Krause, A., et al. (2011). Lyme borreliosis: clinical case definitions for diagnosis and management in Europe. Clin. Microbiol. Infect. 17, 69-79. doi: 10.1111/j.1469-0691.2010.03175.x

Stanek, G., and Reiter, M. (2011). The expanding Lyme Borrelia complexclinical significance of genomic species? Clin. Microbiol. Infect. 17, 487-493. doi: 10.1111/j.1469-0691.2011.03492.x

Stewart, P. E., Wang, X., Bueschel, D. M., Clifton, D. R., Grimm, D., Tilly, K., et al. (2006). Delineating the requirement for the Borrelia burgdorferi virulence factor OspC in the mammalian host. Infect. Immun. 74, 3547-3553. doi: 10.1128/iai.00158-06

Tilly, K., Rosa, P. A., and Stewart, P. E. (2008). Biology of infection with Borrelia burgdorferi. Infect. Dis. Clin. North Am. 22, 217-234. doi: 10.1016/j.idc.2007.12.013

Vandenesch, A., Turbelin, C., Couturier, E., Arena, C., Jaulhac, B., Ferquel, E., et al. (2014). Incidence and hospitalisation rates of Lyme borreliosis, France, 2004 to 2012. Euro. Surveill. 19:20883. doi: 10.2807/1560-7917.ES2014.19.34.20883

Wikel, S. (2013). Ticks and tick-borne pathogens at the cutaneous interface: host defenses, tick countermeasures, and a suitable environment for pathogen establishment. Front. Microbiol. 4:337. doi: 10.3389/fmicb.2013.00337

Wu, Q., Liu, Z., Wang, J., Li, Y., Guan, G., Yang, J., et al. (2013). Pathogenic analysis of Borrelia garinii strain SZ isolated from Northeastern China. Parasit. Vectors 6:177. doi: 10.1186/1756-3305-6-177

Zung, J. L., Lewengrub, S., Rudzinska, M. A., Spielman, A., Telford, S. R., and Piesman, J. (1989). Fine structural evidence for the penetration of the Lyme disease spirochete Borrelia burgdorferi through the gut and salivary tissues of Ixodes dammini. Can. J. Zool. 67, 1737-1748.

Conflict of Interest Statement: The authors declare that the research was conducted in the absence of any commercial or financial relationships that could be construed as a potential conflict of interest.

Copyright (c) 2018 Sertour, Cotté, Garnier, Malandrin, Ferquel and Choumet. This is an open-access article distributed under the terms of the Creative Commons Attribution License (CC BY). The use, distribution or reproduction in other forums is permitted, provided the original author(s) and the copyright owner(s) are credited and that the original publication in this journal is cited, in accordance with accepted academic practice. No use, distribution or reproduction is permitted which does not comply with these terms. 